\title{
Nutzung des Ultraschalls in der Schlachtleistungsprüfung und Zuchtwertschätzung für Schafe
}

\begin{abstract}
Title of the paper: Using of Ultrasound for estimation of carcass composition and prediction of breeding value for sheep's

In Thuringia the subjective visual conformation scores of carcasses for blade (BL), leg (K), back (R), fat overlay (OF) and kidney fat (NF) were added by ultrasonic muscle (USK) and fat (USF) depths of living lambs. Heritabilitity estimates were carried out on 2654 slaughtered and 3228 living and ultrasonic-tested Merinolongwool lambs. Estimates of the subjective conformation scores were very low with 0.178 (BL); 0.143 $(\mathrm{K}) ; 0.165(\mathrm{R})$ and $0.118(\mathrm{OF})$. Slightly higher heritabilities could be estimated for NF (0.234), USK (0.26) and USF (0.174). The genetic correlations between conformation scores for muscle on one hand and OF and USF on the other hand were between 0.313 and 0.848 . In contrast, the genetic correlation between USK on one hand and OF and USF on the other hand was $r_{A}=0.022$ and $r_{A}=-0.110$, respectively). This may increase efficiency of breeding . By means of selection differences, it is recommended to use USK instead of blade and back, and USF instead of fat overlay in selection index.
\end{abstract}

Key Words: sheep, carcass, ultrasound, heritability

\section{Zusammenfassung}

In Thüringen wurde die subjektive Schlachtkörperbonitur für Blatt (BL), Keule (K), Rücken (R) und Oberflächenfett (OF) sowie die gewogene Nierenfettmenge (NF) durch die Ultraschallwerte für Kotelettdicke (USK) und Rückenfettauflage (USF) am lebenden Tier ergänzt. Die Parameterschätzung an 2654 geschlachteten und 3228 lebenden Tieren mit Ultraschallwerten der Rasse Merinolangwollschaf ergab für die subjektiven Boniturnoten sehr niedrige $h^{2}$ Werte von 0,178; 0,143; 0,165 und 0,118 für BL, K, R und OF während für NF, USK und USF 0,234; 0,260 und 0,174 ermittelt wurden. Die Boniturnoten für Bemuskelung wiesen züchterisch ungünstige genetische Korrelationen zum OF und USF zwischen 0,313 und 0,848 auf. Im Gegensatz dazu war die USK von der USF und dem OF kaum beeinflusst $\left(\mathrm{r}_{\mathrm{g}}=0,022 ;-0,110\right)$. Damit ergeben sich verbesserte züchterische Möglichkeiten. Anhand der Selektionsdifferenzen nach der BLUP-Zuchtwertschätzung wird vorgeschlagen, im bisherigen Index Blatt und Rücken durch USK und Oberflächenfett durch USF zu ersetzen.

Schlüsselwörter: Schaf, Schlachtkörper, Ultraschall, Heritabilität

\section{Einleitung}

Ultraschallwerte lebender Tiere haben im letzten Drittel des vergangenen Jahrhunderts eine breite Anwendung gefunden. In den 80iger Jahren erreichten die Geräte eine Qualität, die den Einsatz beim bewollten Schaf ermöglichten (BEACH u.a., 1983). Die Eignung der Ultraschallmesswerte zur Bewertung der Muskelausformung und der chemischen Schlachtkörperzusammensetzung bestätigten unter anderem KRAUTH, 1987; RAMSEY u.a., 1991; XIAOJUN, 1993; MÜLLER, 1995.

Dabei sind die Ergebnisse hinsichtlich der Bedeutung von Fett- und Muskeldicke für den Schlachtkörper durchaus unterschiedlich. Während MÜLLER 1995 anhand seiner Resultate zu dem Schluss kommt, dass der Selektion gegen Fettdicke die wesentliche Bedeutung zukommt, legen die Befunde von PUNTILA u.a. (2002) nahe, auch die 
Muskeldicke zu beachten. Grundsätzlich sollten beide Werte erfasst und hinsichtlich ihres Nutzens in der Selektion untersucht werden.

Abweichend von anderen Schafzuchten liegen in Thüringen nur subjektive Benotungen und die Nierenfettmenge der geschlachteten Tiere zur Auswertung vor. Anschnitte an den Schlachtkörpern waren und sind aus technischen und wirtschaftlichen Gründen nicht durchführbar. Deshalb kommt hier den Ultraschallwerten eine wesentliche Bedeutung zur Schlachtwertbestimmung auch am später geschlachteten Tier zu.

\section{Material}

Das zur Verfügung stehende Datenmaterial umfasst die Ergebnisse der Leistungsprüfung der Jahrgänge 1992-2004 in der Stationen Mühlhausen und Schöndorf des Thüringer Lehr- Prüf- und Versuchsgutes (Tab. 1). Im Rahmen dieser Stationsprüfung erfolgte die Erfassung folgender Leistungen:

Zunahme in der Aufzucht bis zur Anlieferung auf Station ZA (g/Tag)

Prüftagszunahme PTZ (g/Tag)

Lebendmasse Prüfende (am Tag der Ultraschallmessung) USM (kg)

Ultraschall Kotelettmuskeldicke USK (mm)

Ultraschall Rückenfettdicke USF (mm)

Schlachtkörperwarmmasse SKM (kg)

Schlachtkörperbonitur Blatt $\quad$ BL (1-9)

Schlachtkörperbonitur Rücken $\mathbf{R}$ (1-9)

Schlachtkörperbonitur Keule K (1-9)

Schlachtkörperbonitur Oberflächenfett OF (1-9)

Nierenfettmenge NF (g)

Die Prüfgruppen bestehen aus acht bis zwanzig männlichen Tieren. Sie werden im Gewichtsbereich 18 bis $22 \mathrm{~kg}$ angeliefert. Zur Prüfung sind nur Lämmer mit rassetypischen Tageszunahmen in der Aufzuchtphase und rassetypischen Merkmalen zugelassen.

Die Prüfung wird in Gruppenbuchten auf Tiefstreu durchgeführt. Die zur Schlachtkörperbewertung ausgewählten Tiere werden zwischen 38 und $42 \mathrm{~kg}$ Lebendgewicht nüchtern der Schlachtung zugeführt (Landschafrassen zwischen 35 und 37 kg).

Die Fütterung der Prüftiere erfolgte ad libitum mit Lämmermastpellets. Zusätzlich wurden ca. 300g Heu je Tier und Tag verabreicht. Wasser zur freien Aufnahme stand aus Selbsttränken zur Verfügung. Etwa 50\% der eingestallten Tiere wurden nach dem ersten Selektionsentscheid geschlachtet.

Von der Auswertung ausgeschlossen wurden alle Tiere mit einer Prüfdauer unter 22 Tagen, Lämmer mit offensichtlichen Entwicklungsstörungen oder Zunahmen, die mehr als 3,5 Standardabweichungen unter dem Jahresmittel lagen.

Von allen Prüftieren sind die Eltern bekannt. Das Pedigree wurde, wenn verfügbar, bis zur vierten Generation aus den Herdbuchdaten aufgefüllt.

Die Mastleistung wird als Prüftagszunahme ausgewiesen; für die Ermittlung des Schlachtwertes wurden entsprechend der Festlegungen der Zuchtleiter die Nierenfettmenge und die subjektive Bewertung von Keule, Blatt, Rücken und Oberflächenfett genutzt. Seit 1995 liegen von allen geprüften Tieren die Ultraschallwerte vor. 
Tabelle 1

Verfügbare Tierzahlen nach Rassen (Number of animals across breeds)

\begin{tabular}{|c|c|c|}
\hline Rasse & Merkmal & $\mathrm{N}$ \\
\hline Merinoland & Zunahme & 470 \\
\hline \multirow[t]{2}{*}{$(\mathrm{ML})$} & Ultraschall & 425 \\
\hline & Schlachtung & 230 \\
\hline Merinolangwoll & Zunahme & 5118 \\
\hline \multirow[t]{2}{*}{ (MLW) } & Ultraschall & 3228 \\
\hline & Schlachtung & 2654 \\
\hline Rhönschaf & Zunahme & 123 \\
\hline \multirow[t]{2}{*}{$(\mathrm{RH})$} & Ultraschall & 12 \\
\hline & Schlachtung & 87 \\
\hline Schwarzköpfiges & Zunahme & 210 \\
\hline Fleischschaf & Ultraschall & 197 \\
\hline$(\mathrm{SKF})$ & Schlachtung & 91 \\
\hline Leineschaf & Zunahme & 532 \\
\hline \multirow[t]{2}{*}{$(\mathrm{LEI})$} & Ultraschall & 509 \\
\hline & Schlachtung & 376 \\
\hline Suffolk & Zunahme & 159 \\
\hline \multirow[t]{2}{*}{$(\mathrm{SU})$} & Ultraschall & 133 \\
\hline & Schlachtung & 58 \\
\hline Coburger Fuchsschaf & Zunahme & 46 \\
\hline \multirow[t]{2}{*}{$(\mathrm{CF})$} & Ultraschall & 46 \\
\hline & Schlachtung & 24 \\
\hline \multirow[t]{3}{*}{ ML*MLW } & Zunahme & 563 \\
\hline & Ultraschall & 0 \\
\hline & Schlachtung & 241 \\
\hline
\end{tabular}

\section{Die Größenverteilung der väterlichen Halbgeschwistergruppen zeigt Tabelle 2.}

Tabelle 2

Größenverteilung väterlicher Halbgeschwistergruppen (Sizing of half-sib groups)

\begin{tabular}{llll}
\hline $\begin{array}{l}\text { Anzahl } \\
\text { Halbgeschwister }\end{array}$ & \multicolumn{2}{c}{ Anzahl Halbgeschwistergruppen } \\
& Zunahme & Schlachtung & Ultraschall \\
\hline 1 & 39 & 54 & 35 \\
2 & 16 & 36 & 14 \\
$3-5$ & 44 & 92 & 49 \\
$6-10$ & 113 & 127 & 100 \\
$11-20$ & 149 & 101 & 57 \\
$21-40$ & 85 & 27 & 6 \\
$>40$ & 27 & 2 & \\
\hline
\end{tabular}

Tabelle 3 zeigt im Überblick die auswertbaren Tierzahlen und die zugehörigen statistischen Maßzahlen. 
Tabelle 3

Anzahl Beobachtungen [n], Mittelwerte $[\bar{x}$ ], Standardabweichung [s], Schiefe und Exzess für die untersuchten Merkmale (Number of observations [n], means $[\bar{x}]$, standard deviation [s], skewness and excess for analysed traits)

\begin{tabular}{llllll}
\hline & $\mathrm{N}$ & $\bar{x}$ & $\mathrm{~s}$ & Schiefe & Exzess \\
\hline ZA & 7215 & 333,90 & 75,49 & 0,64 & 1,46 \\
PTZ & 7278 & 383,50 & 69,54 & 0,04 & 0,50 \\
USM & 4518 & 40,27 & 2,46 & 0,42 & 2,03 \\
USK & 4694 & 26,39 & 2,79 & 0,59 & 1,87 \\
USF & 4673 & 2,32 & 0,98 & 1,44 & 3,84 \\
SKM & 3765 & 19,07 & 1,99 & 0,41 & 1,83 \\
BL & 3765 & 6,69 & 0,89 & $-0,34$ & 0,59 \\
K & 3765 & 6,91 & 0,87 & $-0,13$ & 0,16 \\
R & 3766 & 6,74 & 1,06 & $-0,37$ & 0,21 \\
OF & 3787 & 6,49 & 0,74 & $-0,31$ & 0,18 \\
NF & 3812 & 204,90 & 95,58 & 1,93 & 8,69 \\
\hline
\end{tabular}

Von 7278 Bocklämmern sind ca. 50\% geschlachtet worden. Die Schlachtung der Lämmer sollte bei einem Gewicht von $40 \mathrm{~kg}$ erfolgen. Damit verbunden wäre eine niedrige Varianz der Schlachtkörpermasse. Leider stand im Laufe der letzten Jahre nicht immer ein zuverlässiger Partner zur Schlachtung zur Verfügung bzw. lagen die Schlachtstätten in größeren Entfernungen, so dass dieses Ziel nicht immer erreicht wurde. Der Variationskoeffizient von 22,9 \% liegt deshalb relativ hoch. Für die Boniturnoten werden Standardabweichungen von einem Punkt erreicht. Beim Oberflächenfett wurde die höchste Note für den magersten Schlachtkörper vergeben. Das Nierenfett zeigt mit einem Variationskoeffizient von ca. 50\% eine erhebliche Schwankungsbreite, deren Ursachen insbesondere in den Abweichungen im hohen Bereich liegen. Das findet seine Bestätigung in dem ausgeprägten Exzess-Wert und der starken Rechtsschiefe. Die Zunahmeleistung während der Aufzuchtphase (Geburt bis zur Einstallung in die Station) wird ebenfalls in die Untersuchungen mit einbezogen, da auch dieser Lebensabschnitt von wirtschaftlicher Bedeutung ist. Ausgespart bleibt die Umstellungsphase zwischen Einstallung und Prüfbeginn.

Etwa die Hälfte der US-geprüften Tiere wurde anschließend geschlachtet und die Schlachtkörper bonitiert. Wegen der geringen Tierzahlen in den anderen Rassen wurden für die Parameterschätzung nur das Merinolangwoll-Material herangezogen.

Wesentliche Selektionsentscheide fallen bei den Lämmern vor der Schlachtung nach der Ultraschallbewertung. Im Datenmaterial sind 2131 Jungböcke enthalten, die noch keine geprüften Nachkommen haben. An diesen sind die realisierten Selektionsdifferenzen zu überprüfen.

Eine weitere Entscheidung fällt mit der Auswahl der Bockväter für die Erzeugung der nächsten Bockgeneration. Hier kommen auch Väter mit Nachkommen in die engere Auswahl. Deshalb wird eine weitere Prüfung an Böcken mit mindesten 8 US geprüften Nachkommen durchgeführt. 232 Böcke stehen hierfür zur Verfügung.

\section{Methoden}

Mögliche fixe Einflussfaktoren für die Stationsmerkmale sind Saisonklasse, Rasse, Geburtstyp und Herkunftsbetrieb. Dabei wurden für die Ultraschallmesswerte das Messdatum und für die Schlachtleistungsmerkmale der Termin der Schlachtung he- 
rangezogen. Die Gruppengröße am Schlachttag schwankte meist zwischen 30 und 80 Tieren je Schlachtdatum. Die Ultraschallmessung erfolgte an etwa doppelt so vielen Tieren je Messtag. Waren die Gruppen kleiner 10, wurden sie innerhalb eines Monats zusammengefasst. Für die Prüftagszunahme war das Quartal des Prüfbeginns als zeitlicher Faktor ausschlaggebend. In fast allen Betrieben wird nur jeweils eine Rasse gehalten. In Betrieben mit verschiedenen Rassen werden diese in der Regel in getrennten Herden geführt, so dass Betriebs- und Rasseeffekte nicht zu trennen sind. Deshalb werden die Betriebe innerhalb der Rassen getestet.

Für die Schlachtleistungsmerkmale bestand darüber hinaus die Notwendigkeit, die Berücksichtigung der Schlachtkörpermasse als Covariable zu prüfen. Die Signifikanzen wurden mit GLM-Prozeduren (SPSS) in einem Modell mit allen Faktoren aber ohne Tiereffekt geprüft.

Mit den Ergebnissen dieser Voruntersuchungen war das Modell zur Schätzung der genetischen und umweltbedingten Varianz- und Covarianzmatrizen aufzubauen. Die REML-Schätzung erfolgte mit dem VCE-Paket, Version 5.1 bei Einsatz des analytischen Gradienten. Zur Zuchtwertschätzung wurde das Programmpaket Pest 3.1. genutzt.

Für die jungen Bocklämmer mit Ultraschalluntersuchung erfolgt eine simulierte Selektion mit 1/3 Selektionsanteil. Mit den Ultraschallwerten ergeben sich neue Möglichkeiten. Demzufolge ist der Ersatz des derzeitigen Teilzuchtwertes Bemuskelung durch neue Varianten zu prüfen. Folgende Varianten werden im folgenden verglichen:

1. Die bisherigen Teilzuchtwerte Bemuskelung ( $3 *$ Keule $+2 *$ Rücken $+1 *$ Blatt) und Fett ( $2 *$ Oberflächenfett $+1 *$ Nierenfett) ohne Nutzung des Ultraschalls

2. Der bisherige Teilzuchtwert wie Variante 1 mit Nutzung des Ultraschalls als Informationsmerkmal.

3. Ersatz der Wichtung der Bemuskelungsnoten für Rücken und Blatt durch die Ultraschall-Muskeldicke, Ersatz der Note für Oberflächenfett durch den Ultraschallwert für Fett.

Die Teilzuchtwerte werden entsprechend der Vorgaben der Zuchtleiter zum Gesamtzuchtwert ( $5 *$ Bemuskelung+5*Verfettung+2*Zunahme) zusammengefasst.

Ebenso erfolgt diese Einteilung für die Böcke mit Nachkommen. Auf ein Splitten der Halbgeschwistergruppen wird verzichtet. Bei so geringen Tierzahlen werden die Halbgeschwistergruppen zu klein und die Ergebnisse so unsicher, dass ein so gewonnenes Ergebnis keine weiterreichenden Schlussfolgerungen zulassen würde.

\section{Ergebnisse}

Die Tabelle 4 zeigt die genutzten Modelle. Abgesehen vom Geburtstyp erwiesen sich die jeweils getesteten Faktoren durchgängig als signifikant. Die Ergebnisse zu den Ultraschallwerten waren in dieser Form zu erwarten und entsprechen den Ergebnissen der Boniturmerkmale.

Eine Überprüfung anhand phänotypischer Regressionsfunktionen führte zum Schluss, dass eine lineare Berücksichtigung der Körpermasse für die Ultraschallwerte ausreicht. Die mit diesen Modellen multivariat ermittelten Parameter enthält Tabelle 5.

Die Standardfehler der Heritabilitäten liegen zwischen 0,014 und 0,029; die der genetischen Korrelationen zwischen 0,023 und 0,092 und die der Umweltkorrelationen zwischen 0,012 und 0,027. 
Tabelle 4

Signifikanzen der geprüften Einflussfaktoren (Significances of effects on conformation scores and ultrasonic measurements)

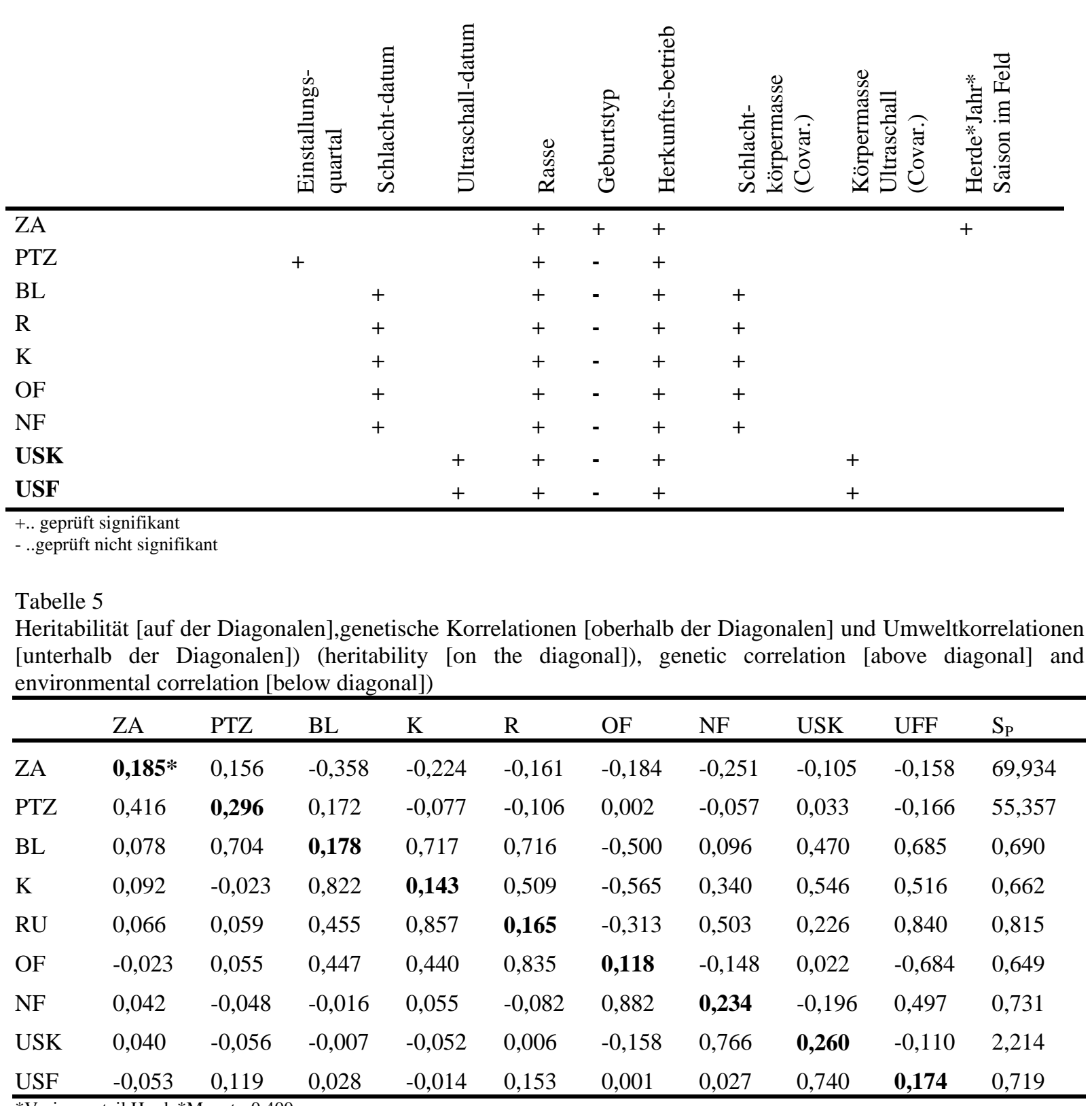

*Varianzanteil Herde*Monat $=0,400$.

Die Schätzwerte zeigen das in Thüringen bestehende Problem der niedrigen Heritabilitäten für die Merkmale der Schlachtkörperbonitur.

Andererseits macht die vergleichsweise höhere Heritabilität der Ultraschallmesswerte die Eignung des Ultraschalls für die Erfassung der Fleischigkeit am lebenden Tier deutlich. Der prinzipielle Unterschied zwischen den Benotungen und dem Ultraschall besteht in den genetischen Korrelationen zu den Fettmerkmalen. Während die Noten sehr hohe genetische Korrelationen zu den Fettwerten aufweisen, sind diese für die Ultraschall-Muskeldicke nicht vorhanden. Hier sind auch die Ursachen für die nicht sehr hohen genetischen Korrelationen zwischen den Bemuskelungs-Noten und der Ultraschall-Muskeldicke zu suchen. Insbesondere die genetische Korrelation zur Rückenmuskelnote fällt deutlich niedriger als erwartet aus. Dabei ist beachtenswert, dass besonders die Rückenmuskelnote die im züchterischen Sinne negativ zu bewertenden 
genetischen Korrelationen zu den Verfettungswerten am deutlichsten zeigt. Während die Boniturnoten eine vom Fett deutlich beeinflusste genetische Varianz zeigen, besteht dieses Problem für den Ultraschall nicht.

Die Umweltkorrelationen zwischen den Fleisch und Fettwerten liegen dagegen vorwiegend um Null. Für die Fettauflage war nur eine geringe Steigerung der Heritabilität durch Einführung des Ultraschalls zu erreichen. Die Ursache dürfte in erster Linie in der Messgenauigkeit des Gerätes liegen. Die Skala der Messwerte beträgt 1mm. Bei einem Mittelwert von 2 bis 3mm und einer Standardabweichung von etwa einem Millimeter ist dieses Raster zu grob. Die hohe genetische Korrelation zur Bonitur am Schlachtkörper bestätigt aber die prinzipielle Eignung des Verfahrens auch zur Bestimmung der Fettauflage.

Die Beziehungen zwischen Zunahmeleistungen und den Schlachtleistungsmerkmalen bleiben durchgängig niedrig.

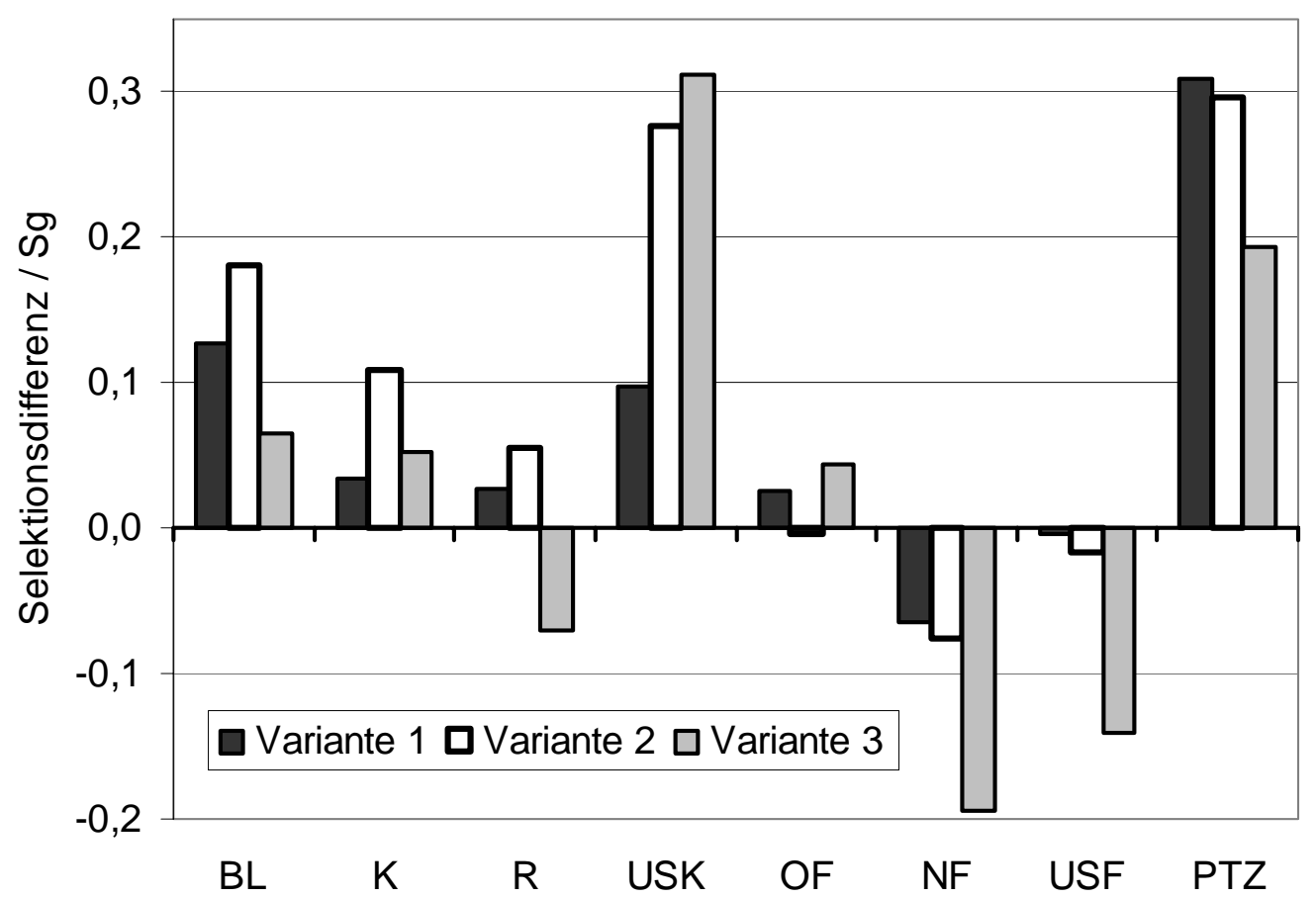

Abb. 1: Selektionsdifferenzen in genetischen Standardeinheiten nachkommengeprüfter Böcke bei Selektion nach verschiedenen Varianten, Selektionsanteil 30\% 1... ohne Ultraschall 2... Ultraschall als Informationsmerkmal 3... Ultraschall mit eigenem wirtschaftlichen Gewicht (selection differences in genetic standard deviation of ultrasonic measured progeny tested rams by selection with alternative Indices, $30 \%$ selection quota; 1 ... without ultrasound measurement, $2 \ldots$ ultrasound measure as information attribute $3 \ldots$ ultrasound measure with own economical weighting)

Selektionsdifferenzen bei simulierter Selektion mit $30 \%$ Selektionsanteil sind in der Abbildung 1 für Böcke mit mindestens 8 US-getesteten Nachkommen, von denen im Mittel 4 geschlachtet wurden, dargestellt. Ohne Ultraschall (Variante 1) sind auch bei Nachkommenprüfung für den Schlachtkörper kaum Fortschritte zu erzielen (Abb. 1). Hierfür sind zwei Ursachen verantwortlich: Die niedrige Heritabilität der Boniturnoten lässt bei der in der Schafzucht anzutreffenden niedrigen Zahl geprüfter Nachkommen keine genauen Zuchtwerte zu und die hohen, züchterisch ungünstigen genetischen Korrelationen zwischen Fettmerkmalen und Boniturnoten engen den Selektionsspielraum für den Schlachtkörper insgesamt ein. Der Index weicht auf hohe Zunahmeleistung aus. Dies wäre auch mit noch höheren Gewichten für die Schlachtkörpermerkmale nicht zu vermeiden. Allein die Nutzung des Ultraschalls als Informationsmerk- 
mal (Variante 2) bringt für die Muskelausformung einen erheblichen Fortschritt, für die Verfettung bleiben die Ergebnisse aber unbefriedigend. Erst eine Wichtung des Ultraschalls (Variante 3) bringt hier Verbesserungen, die für das Nierenfett deutlich, für die Oberflächen-Note aber gering ausfällt. Aufgrund der engen genetischen Korrelationen muss dann in Kauf genommen werden, dass die Boniturnoten für die Muskelausformung nur gering verbessert werden können, während die Ultraschallwerte sowohl für Fett als auch für Muskeldicke deutlich verbesserbar sind.

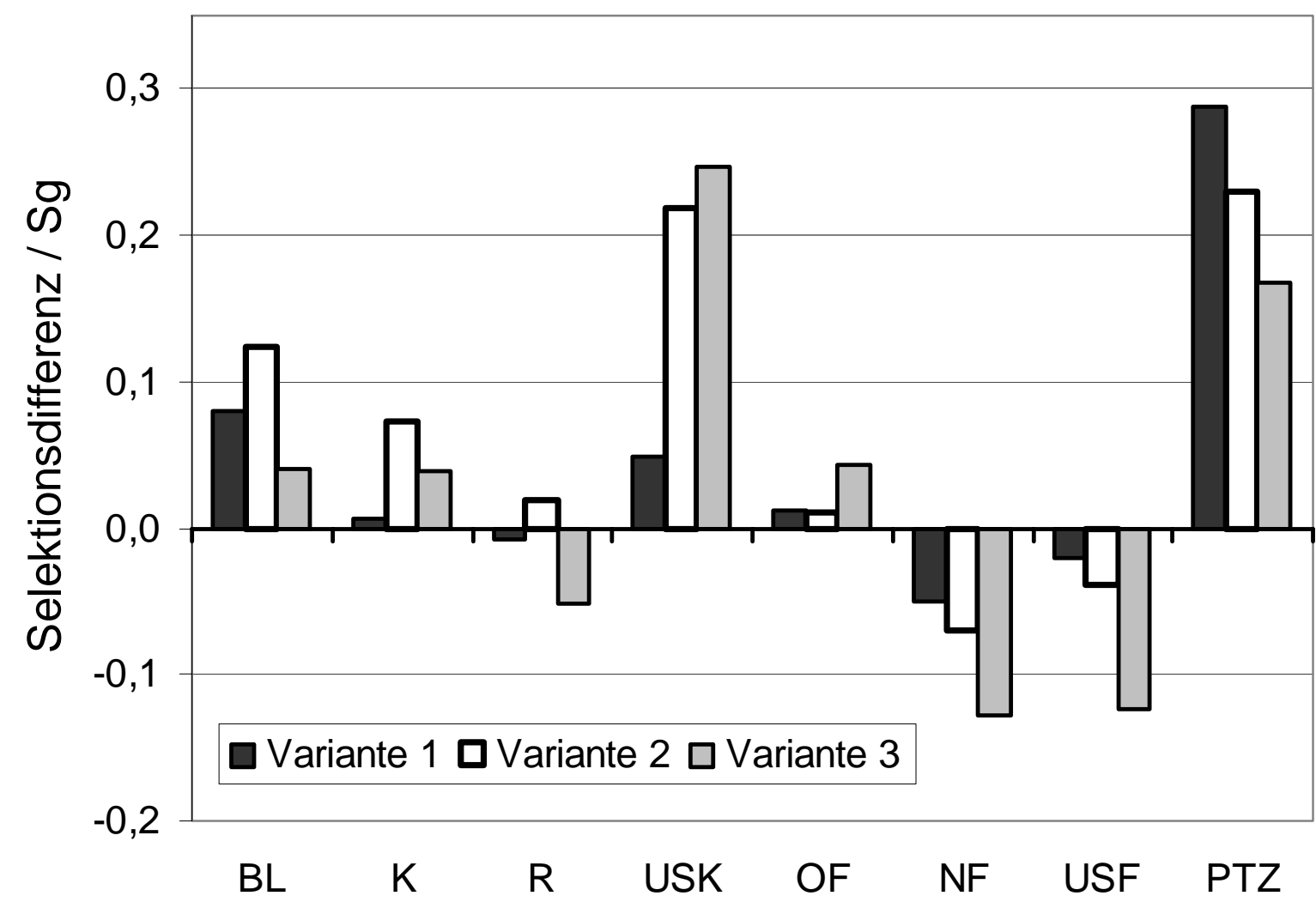

Abb. 2: Selektionsdifferenzen in genetischen Standardeinheiten ultraschallgeprüfter Jungböcke bei Selektion nach verschiedenen Varianten, Selektionsanteil 30\% 1... ohne Ultraschall 2... Ultraschall als Informationsmerkmal 3... Ultraschall mit eigenem wirtschaftlichen Gewicht (selection differences in genetic standard deviation of ultrasonic measured young rams by selection with alternative Indices, $30 \%$ selection quota; 1 ... without ultrasound measurement, 2... ultrasound measurement as information attribute 3... ultrasound measurement with own economical weighing)

Prinzipiell zeigt sich das gleiche Bild bei den Jungböcken (Abb. 2), die allein aufgrund der Vorfahrenzuchtwerte und der Eigenleistung selektiert werden. Auch die Dimension der Selektionsdifferenzen bleibt kaum hinter den nachkommengeprüften zurück!

\section{Diskussion}

Grundsätzlich sollte sich ein Zuchtziel an den zukünftigen wirtschaftlichen Gegebenheiten orientieren. Dies verlangt die Berücksichtigung der Produktionskosten ebenso wie die Anforderungen des Marktes. Mit dem Grenznutzen können beide Faktoren zusammengeführt werden. Leider werden in der deutschen Schafvermarktung Qualitätsunterschiede nur sehr pauschal und grob bewertet, sodass Anforderungen der Verbraucher an die Schlachtkörperqualität, die CARPENTER schon 1966 quantifizierte, den Erzeuger nur allgemein und verbal erreichen. Auch Art und Umfang der Lammvermarktung lassen eine Einführung automatisierter Schlachtkörperbewertun- 
gen, die sich als durchaus brauchbar erwiesen haben (BARWICK, 1980; GARRET u.a., 1992; BRADY u.a., 2003; CUNHA u.a., 2004), vorläufig in Deutschland nicht erwarten. Deshalb wird eine Qualitätsdifferenzierung in der Regel weiterhin nur partieweise erfolgen. Daraus ergibt sich das Risiko, dass Erzeuger zu der Auffassung gelangen, Schlachtkörperqualität sei sekundär. Dem muss permanent entgegen gearbeitet werden, da deutsches Lammfleisch ständigem Konkurrenzdruck ausländischer Anbieter und anderer Fleischarten ausgesetzt ist und das Image minderer Qualität beim Verbraucher langfristig verheerende Folgen hätte.

Auch in der Zucht versucht man mit einer hohen Bewertung der Schlachtkörperqualität dem entgegen zu wirken. Nur für wenige deutsche Rassen liegen allerdings Untersuchungen zu Beziehungen zwischen Hilfsmerkmalen der Schlachtkörperbewertung und dem Marktwert der Schlachtkörper vor (MÜLLER, 1995; QUANZ, 1993) . Deshalb beschränkt sich die Zuchtzielformulierung nach wie vor auf eine empirische Vorgabe, die je nach Bedingung und Möglichkeit umgesetzt wird. In Thüringen konnten zur Bewertung der Schlachtkörper nur die subjektiven Noten und das Nierenfett herangezogen werden. Leider sind die für die subjektiven Noten berechneten $\mathrm{h}^{2}$-Werte wie bei KEFELEGN u.a. (1998) sehr niedrig und die genetischen Korrelationen zwischen Muskel- und Fettbonitur sehr ungünstig. Dies kann nicht einfach damit erklärt werden, dass runde Schlachtkörper vom Bonitierenden in beiden Merkmalskomplexen als dicker angesehen werden, denn die Restvarianzen sind für beide Merkmalskomplexe deutlich höher, die Rest-Covarianzen tendieren aber gegen Null. Über ähnliche Probleme berichten KEMPSTER u.a. (1982), GARRET u.a. (1992) und JONES u.a. (1992). Die Nutzung einer Bonitur zur Schlachtkörperbewertung beim Lamm wird deshalb vollständig in Frage gestellt (KEMPSTER u.a., 1982) oder ihr eine eher nur geringe Bedeutung zugemessen (JONES u.a., 1992).

Unter diesen Bedingungen stellt die Ultraschalluntersuchung eine wesentliche Verbesserung der züchterischen Möglichkeiten dar. Die hierfür geschätzten Heritabilitäten bewegen sich zwar unterhalb derer von PUNTILA u.a. (2002) oder RODEN u.a. (2003), sind aber mit denen von MANIATIS und POLLOT (2002) oder DEWI u.a. (2002) vergleichbar. Alle diese Autoren fanden in Übereinstimmung mit den hier ermittelten Ergebnissen für die Muskeldicke höhere $\mathrm{h}^{2}$-Werte als für Fettdicke, während SAVAS u.a. (2001) für Muskeldicke mit 0,14 eine sehr niedrige Heritabilität, für Fettdicke mit 0,38 aber eine eher hohe schätzten.

Im Gegensatz zu Tierarten mit entwickelter Schlachtkörperbewertung ermöglicht die Nutzung des Ultraschalls beim Schaf nicht nur den verbesserten Selektionsentscheid aufgrund einer Eigenleistung am lebenden Tier, er führt unter den vorliegenden Bedingungen auch zu einer Verbesserung der Genauigkeit der Schlachtkörperbewertung später geschlachteter Tiere. Der wesentliche Vorteil der Ultraschallwerte in dem untersuchten Tiermaterial besteht aber in der ,Entkopplung' von Fett und Fleisch.

Problematisch bleibt dagegen, dass die tatsächlichen genetischen Beziehungen zwischen allen hier nutzbaren Schlachtkörpermerkmalen und dem ökonomischen Schlachtkörperwert wie handelsfähige Fleischmenge nicht wirklich bekannt sind und nur auf allgemeinen Mutmaßungen beruhen. Auch hier liegt aber die Vermutung nahe, dass die messbaren Ultraschallwerte und der Nierenfettanteil dem näher kommen, als die subjektiven Boniturnoten (QUANZ, 1993; MÜLLER, 1995; KEMPSTER u.a., 1982; GARRET u.a., 1992; JONES u.a., 1996; RAMSEY u.a., 1991). 
Aus den genetischen Parametern ergibt sich zwangsläufig, dass die erzielten Selektionsdifferenzen der Jungböcke bei gleichen Selektionsanteilen kaum hinter denen der nachkommengeprüften Tiere zurückbleiben. Mit der Selektion der Jungböcke werden deutlich höhere Selektionsintensitäten möglich. Voraussetzung für die stärkere Orientierung auf eine Eigenleistungsprüfung auf Station ist die Beschickung der Prüfstation zu hohen Anteilen mit potentiellen Zuchtböcken. Dies geschieht in Thüringen zum Teil, ist aber noch ausbaufähig. Damit wäre ein wesentlich höherer Zuchtfortschritt realisierbar.

\section{Literatur}

BARWICK, S.A:

Carcass Measurements and their associating with yield of cuts and tenderness in lamb. Wool Technol. Sheep Breed 28(1980), 19-23

BEACH, A.D.; TUCK, D.L.; TWIZELL, R.J.:

Ultrasonic equipment for the measurement of backfat on unshorn sheep. Ultrasonics 21 (1983), 187-187

BRADY, A.S.; BELK, K.E.; LEVALLEY, S.B.; DALSTED, N.L.; SCANGA, J.A.; TATUM, J.D.; SMITH, G.C.:

An evaluation of the lamb vision system as a predictor of lamb carcass red meat yield percentage. J. Anim. Sci. 81 (2003), 1488-1498

CARPENTER, Z.L.:

What is the consumer-preferred lamb? J. Anim. Sci. 25 (1966), 1232-1235

CUNHA, B.C.N.; BELK, K.E.; SCANGA, J.A.; LEVALLEY, S.B.; TATUM, J.D.; SMITH, G.C.:

Development and validation of equations utilizing lamb vision system output to predict lamb carcass fabrication yields. J. Anim. Sci. 82 (2004), 2069-2076

DEWI, I.A.; SAATCI, M.; ULUTAS, Z.:

Genetic parameters of weights, ultrasonic muscle and fat depths, maternal effect and reproductive traits in Welsh Mountain sheep. Anim Sci. 74 (2002), 399-408

GARRET, R.P.; EDWARDS, J.W.; SAVELL, J.W.; TATUM, J.D.:

Evaluation of the Hennesey grading probe to predict yields of lamb carcasses fabricated to multiple end points. J. Anim. Sci 70 (1992), 1146-1152

JONES, S.D.M.; JEREMIAH, L.E.; TONG, A.K.W.; ROBERTSON, W.M.; GIBSON, L.L.:

Estimation of lamb carcass composition using an electronic probe, a visual system and carcass measurement. Can. J. Anim. Sci. 72 (1992), 237-244

JONES, S.D.M.; ROBERTSON, W.M.; PRICE, M.A.; COUPLAND, T.:

The Prediction of saleable meat yield in lamb carcasses. Can J. Anim. Sci. 76 (1996), 49-53

KEFELEGN, K.; SÜß, R.;MIELENZ, N.; SCHÜLER, L.; LENGERKEN, G. v.:

Schätzung genetischer Parameter für Merkmale der Mastleistung und des Schlachtkörperwertes beim Schaf. Arch. Tierz., Dummerstorf 41 (1998), 463-472

KEMPSTER, A.J.; CUTHBERTSON, A.; HARRINGTON, G.:

The relationship between conformation and the yield and distribution of lean meat in the carcasses of British pigs, cattle, and sheep. Meat Sci. 6 (1982), 37-53

KRAUTH, R.:

Morphologische Parameter als Hilfsmittel zur Abschätzung der Schlachtkörperzusammensetzung in der Schafzucht unter besonderer Berücksichtigung von Ultraschallmaßen. Diss. Hohenheim (1987)

MANIATIS, N.; POLLOT, G.E.:

Nuclear, cytoplasmatic and environmental effect on growth, fat, and muscle traits in Suffolk lamb from a sire referencing scheme. J. Anim. Sci. 80 (2002), 57-67

MÜLLER, U.:

Planungsrechnungen zur Optimierung von Zuchtsystemen für die Verbesserung der Mastleistung und Schlachtkörperqualität der Rasse Merinofleischschaf unter Verwendung des Ultraschallmeßverfahrens am lebenden Tier. Diss. Leipzig (1995)

PUNTILA, M.L.; MÄKI, K.; RINTALA, O.:

Assessment of carcass composition based on ultrasonic measurement and EUROP conformation class of live lambs. J. Anim. Breed. Gen. 119 (2002): 367-378

RAMSEY, C.B.; KIRTON, A.H.; HOGG, B.; DOBBIE, J.L.:

Ultrasonic, needle, and carcass measurements for predicting composition of lamb carcasses. J. Anim: Sci 96 (1991), 3655-3664

RODEN, J.A.; MERRELL, B.G.; MURRAY, W.A.; HARESIGN, H.: 
Genetic analysis of live weight and ultrasonic fat and muscle traits in a hill sheep flock undergoing breed improvement utilizing an embryo transfer programme. Anim. Sci. 76 (2003), 367-373

SAVAS, T.; RÖHE, R.; KALM, E.:

Genetisch-statistische Analyse der Mastleistungs- und Schlachtkörperwertmerkmale von Fleischschafrassen aus der Feldprüfung von Schleswig-Holstein. Züchtungskunde 73 (2001), 62-73

XIAOJUN, L.:

Selektion zur Verbesserung des Schlachtkörperwertes beim Schaf mit Hilfe der Ultraschalltechnik. Diss. Universität Hohenheim (1993)

Eingegangen: 24.11.2004

Akzeptiert: 08.03.2005

Anschrift der Verfasser

Dr. ERHARD GERNAND, Dr. HEIKE LENZ

Thüringer Landesanstalt für Landwirtschaft Jena, Abt Tierproduktion,

Standort Clausberg

Am Rennsteig 3

99819 OBERELLEN OT Clausberg

E-mail: e.gernand@clausberg.tll.de

E-mail: h.lenz@clausberg.tll.de 